\title{
Traveling-Stripe Forcing Generates Hexagonal Patterns
}

\author{
D. G. Míguez, ${ }^{1}$ E. M. Nicola, ${ }^{2}$ A. P. Muñuzuri, ${ }^{1}$ J. Casademunt, ${ }^{2}$ F. Sagués, ${ }^{3}$ and L. Kramer ${ }^{4}$ \\ ${ }^{1}$ Grupo de Física non Lineal, Universidade de Santiago de Compostela, E-15782 Santiago de Compostela, Spain \\ ${ }^{2}$ Departament d'Estructura i Constituents de la Matèria, Facultat de Física, Universitat de Barcelona, \\ Avinguda Diagonal 647, E-08028 Barcelona, Spain \\ ${ }^{3}$ Departament Química Física, Universitat de Barcelona, Martí i Franqués 1, E-08028 Barcelona, Spain \\ ${ }^{4}$ Physikalisches Institut, Universität Bayreuth, D-95440 Bayreuth, Germany
}

(Received 12 September 2003; published 23 July 2004)

\begin{abstract}
We study the response of Turing stripe patterns to a simple spatiotemporal forcing. This forcing has the form of a traveling wave and is spatially resonant with the characteristic Turing wavelength. Experiments conducted with the photosensitive chlorine dioxide-iodine-malonic acid reaction reveal a striking symmetry-breaking phenomenon of the intrinsic striped patterns giving rise to hexagonal lattices for intermediate values of the forcing velocity. The phenomenon is understood in the framework of the corresponding amplitude equations, which unveils a complex scenario of dynamical behaviors.
\end{abstract}

DOI: $10.1103 /$ PhysRevLett.93.048303

The response of patterns to external forcing provides a tool to probe the inherently nonlinear mechanisms of self-organization under nonequilibrium constraints. During the past few years, attention had mostly focused on resonances or locking of spatially structured states, either oscillatory or stationary, under purely temporal [13] or spatial (steady) modulations [4-7]. We extended these scenarios recently by proposing a particularly simple mode of spatiotemporal forcing [8]. The situation considered was the dynamical response of a striped Turing pattern to a resonant stripe modulation of a control parameter, sweeping the system at small velocities. Without the forcing, the instability leads to a stationary periodic stripe pattern (finite wave number, zero frequency) $[9,10]$.

We report here on the discovery of a surprising symmetry breaking phenomenon in the forced system, leading to the emergence of hexagonal lattices, when the forcing is applied at larger velocities. The experimental context, as it was in our previous work [8], refers to the chlorine dioxide-iodine-malonic acid (CDIMA) reaction [11], which has the interesting feature of being photosensitive [12]. To understand this new phenomenon, the scenario is interpreted within an amplitude equation generic framework.

Experiments.-The experiments were carried out in a thermostated one-feeding chamber continuously feed unstirred reactor maintained at $4 \pm 0.5^{\circ} \mathrm{C}$ using an experimental setup described elsewhere [13]. Reagents of the (photosensitive) CDIMA reaction were continuously pumped into the reactor with feed-stream concentrations: $\left[I_{2}\right]=0.45 \mathrm{mM}, \quad[M A]=1.2 \mathrm{mM}, \quad\left[\mathrm{ClO}_{2}\right]=0.1 \mathrm{mM}$, $\left[\mathrm{H}_{2} \mathrm{SO}_{4}\right]=10 \mathrm{mM}$, and $[P V A]=10 \mathrm{~g} / \mathrm{l}$. Under these conditions and without illumination, the system spontaneously develops labyrinthine patterns with a wavelength of $2 \pi / k_{c}=0.54 \pm 0.02 \mathrm{~mm}$. In our experiments, a periodic array of light stripes with a wavelength of precisely $0.54 \mathrm{~mm}$ (i.e., in spatial resonance) were focused on the
PACS numbers: 82.40.Ck, 47.20.Ky, 47.54.+r, 89.75.Kd

gel layer and were moved perpendicularly to the stripes with a well-controlled and constant velocity $\boldsymbol{v}_{f}$. Light intensity was varied using filters on a $300 \mathrm{~W}$ halogen lamp. Digital images were taken periodically (at 8-9 min intervals) using a charge-coupled device camera.

Considering $v_{f}$ as the control parameter, two trivial limiting cases are easily identified. At sufficiently small velocities, the striped pattern adiabatically follows the imposed light pattern. Such a traveling wave mode [8] is hereafter referred to as a pattern of entrained stripes (ES). Slightly increasing the velocity of the forcing, the stripes continue following the imposed pattern but with a spatially homogeneous and time modulated amplitude. Here we will refer to this pattern as oscillating stripes (OS), rather than modulated traveling waves [8]. Just in the opposite limit, i.e., for large values of $v_{f}$, the system averages out the illumination and exhibits the static pattern corresponding to a uniform illumination with the same mean value (stripes in our case).

Striking new results, those at the core of this Letter, appear for intermediate values of the forcing velocity. In this regime the system responds, quite unexpectedly, organizing itself into a hexagonal lattice and breaking the initial continuous transversal symmetry of the problem. Depending on the light intensity, this lattice may either oscillate or travel. It is worth remarking that this system supports hexagonal patterns but for a completely different range of parameters.

A typical dynamical pattern for high light intensities is shown in Fig. 1. The amplitude of the hexagonal lattice oscillates periodically with a frequency similar to the one imposed by the forcing [see Figs. 1(d) and 1(e)]. This pattern transits between lattices of white and black spots and appears to oscillate coherently around a fixed position. We will refer to this dynamical pattern as oscillating hexagons $(\mathrm{OH})$. Let us remark that this behavior is robust and appears for a wide spectrum of $v_{f}$. This robustness extends in a broad range of light intensities, except for its 


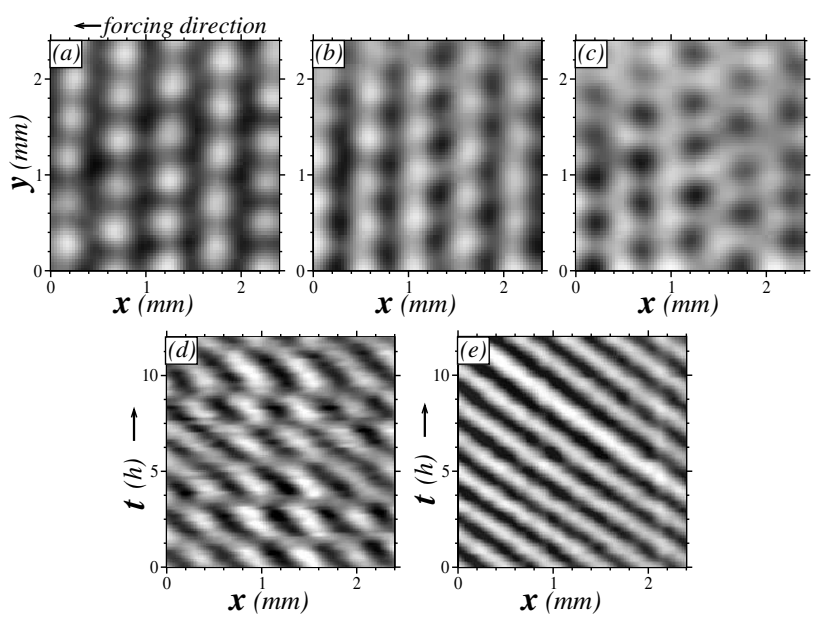

FIG. 1. Oscillating hexagons for a forcing velocity of $v_{f}=$ $0.28 \mathrm{~mm} / \mathrm{h}$. In this case, the pattern exhibits three hexagonal lattices of white spots (a), black spots (c), and an intermediate state (b) where both lattices are interlaced. The corresponding spatiotemporal plot of cuts along the horizontal direction of the pattern and the forcing are shown in (d) and (e), respectively.

lower limit where more complex behavior has been observed. For example, the transition between white and black spot lattices may occur in an abrupt and unpredictable way.

For low intensities, a different kind of hexagonal patterns has been observed in some experiments (see Fig. 2). In contrast to the previous case, this lattice is composed of black spots which move entrained with the forcing [see Figs. 2(b) and 2(c)]. We refer to this spatiotemporal structure as a pattern of entrained hexagons $(\mathrm{EH})$. We note that this spatial arrangement is less ordered than the $\mathrm{OH}$ (i.e., it has a shorter coherence length). It is worth stressing that EH appear only for a small range of forcing velocities.

Amplitude Equations.-Near threshold, where the behavior is expected to be generic, the pattern can be described by a superposition of stripes (or rolls). The forcing is characterized by its wave number, which we here choose to be equal to the critical one $k_{c}$ (1:1 resonance), and the (small) frequency $\omega=v_{f} k_{c}$. We allow for stripes with the wave vector along that of the forcing (with complex amplitude $A$ ) and the addition of two roll systems (with $B_{1}$ and $B_{2}$, respectively) that generate

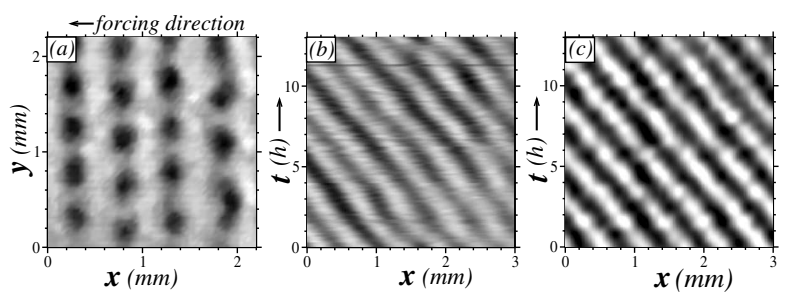

FIG. 2. Entrained hexagons appearing for a forcing velocity of $v_{f}=0.16 \mathrm{~mm} / \mathrm{h}$. In (a) we show a snapshot of the experiment and in (b) and (c) the corresponding spatiotemporal plots of the pattern and forcing, respectively. hexagons (or, according to Busse's designation, "hexarolls" [14]). The wave vectors associated with $A, B_{1}$, and $B_{2}$ are $\vec{k}=k_{c} \hat{x}, \vec{k}_{1}=k_{c}(-\hat{x}+\sqrt{3} \hat{y}) / 2$, and $\vec{k}_{2}=$ $k_{c}(-\hat{x}-\sqrt{3} \hat{y}) / 2$, respectively. The basic assumption is that the forcing has a negligible influence on $B_{1}, B_{2}$, since for those rolls the projected wave vector of the forcing is far from resonance. In this situation, the corresponding amplitude equations can be shown to be

$$
\begin{aligned}
\partial_{t} A= & {\left[\mu-g|A|^{2}-h\left(\left|B_{1}\right|^{2}+\left|B_{2}\right|^{2}\right)\right] A+\alpha B_{1}^{*} B_{2}^{*} } \\
& +\epsilon \exp (-i \omega t), \\
\partial_{t} B_{1}= & {\left[\mu-g\left|B_{1}\right|^{2}-h\left(|A|^{2}+\left|B_{2}\right|^{2}\right)\right] B_{1}+\alpha B_{2}^{*} A^{*}, } \\
\partial_{t} B_{2}= & {\left[\mu-g\left|B_{2}\right|^{2}-h\left(|A|^{2}+\left|B_{1}\right|^{2}\right)\right] B_{2}+\alpha B_{1}^{*} A^{*}, }
\end{aligned}
$$

where we assume $h>g$, so that in the absence of the resonance terms (i.e., for $\alpha=0$ ) stripes are preferred over hexagons.

The explicit time dependence can be eliminated by going into a comoving frame such that $A=\bar{A} \exp -i \omega t$ and $B_{1,2}=\bar{B}_{1,2}$ expi $\omega t / 2$ (the factor $1 / 2$ arises from the projection). Then $\mu$ is replaced in (1a) by $\mu+i \omega$ and in (1b) and (1c) by $\mu-i \omega / 2$. Fixed points in this representation correspond to entrained solutions.

We will here look for solutions with $\left|B_{1}\right|=\left|B_{2}\right|$. It is easy to verify that then the difference between the phases of $B_{1}$ and $B_{2}$ is constant in time. In fact, we can choose $B_{1}=B_{2}(=B)$.

The freedom to specify the scale of $t$ and of $A$ and $B$ allows us to choose the relevant parameters $h^{\prime} \equiv h / g$, $\alpha^{\prime} \equiv \alpha / \sqrt{|\mu| g}, \epsilon^{\prime} \equiv \sqrt{g} \epsilon /|\mu|^{3 / 2}, t^{\prime} \equiv|\mu| t$, and $\omega^{\prime} \equiv$ $\omega /|\mu|$ (together with the sign of $\mu$ ). In this representation, Eqs. (1) take the scaled form (we keep the same symbols for the scaled $A$ and $B$ and consider the case $\mu>0$ )

$$
\begin{aligned}
\partial_{t^{\prime}} \bar{A}= & \left(1+i \omega^{\prime}-|\bar{A}|^{2}-2 h^{\prime}|\bar{B}|^{2}\right) \bar{A} \\
& +\alpha^{\prime} \bar{B}^{* 2}+\epsilon^{\prime}, \\
\partial_{t^{\prime}} \bar{B}= & {\left[1-i \omega^{\prime} / 2-|\bar{B}|^{2}-h^{\prime}\left(|\bar{A}|^{2}+|\bar{B}|^{2}\right)\right] \bar{B} } \\
& +\alpha^{\prime} \bar{B}^{*} \bar{A}^{*} .
\end{aligned}
$$

These equations allow the symmetries: (i) $\left(\bar{B}, \alpha^{\prime}\right) \rightarrow$ $\left(i \bar{B},-\alpha^{\prime}\right)$, which expresses the fact that there are two kinds of hexagon solutions (connected by a shift in $x$ of $2 \pi / k_{c}$ ) that are degenerate only for $\alpha^{\prime}=0$; and (ii) $\bar{B} \rightarrow$ $-\bar{B}$, which corresponds to a shift of the pattern by $2 \pi /\left(\sqrt{3} k_{c}\right)$ along the $y$ direction.

We first consider the case of stripes, i.e., $\bar{B}=0$, where only the parameters $\epsilon^{\prime}$ and $\omega^{\prime}$ are relevant.

Fixed points of (2a) correspond to ES and are solutions of

$$
\left(F^{2}-1\right)^{2} F^{2}+\omega^{\prime 2} F^{2}=\epsilon^{\prime 2},
$$

where $\quad \bar{A}=F e^{i \bar{\theta}} \quad$ and $\quad \sin \bar{\theta}=\left(\omega^{\prime} / \epsilon^{\prime}\right) F=$ $\sqrt{1-\left(F^{2}-1\right)^{2} F^{2} / \epsilon^{12}}$.

For $\epsilon^{\prime 2}<8 / 27 \simeq 0.296$, there is a window of $\omega^{\prime}$ with three real solutions of Eq. (3) where the intermediate 
branch is unstable [see Figs. 3(a) and 3(b)]. These solutions are generated and destroyed pairwise by saddlenode bifurcations. The region of multiple solutions ends in a cusp at the critical point $\omega^{\prime 2}=1 / 3, \epsilon^{\prime 2}=8 / 27$ where Eq. (3) has three identical solutions with $F^{2}=2 / 3$. For $\epsilon^{\prime 2}>8 / 27$, a unique solution exists for all values of $\omega^{\prime}$. In addition, solutions become Hopf unstable (with frequency $\Omega_{O S}=\sqrt{\omega^{\prime 2}-1 / 4}=\sqrt{2 \epsilon^{\prime 2}-1 / 2}$ ) when $F^{2}$ drops below $1 / 2$. This transition, which leads from entrained stripes to oscillating stripes, with a time modulated amplitude [ES $\rightarrow$ OS in the following; see Fig. 3(a) and 3(b)], has been studied in [8]. A detailed stability analysis of the amplitude equation including wave number mismatch will be presented elsewhere.

We now focus on solutions of (2) with $\bar{B} \neq 0$, thus allowing for the formation of hexagons. The first step is to study the stability of entrained stripes ES towards growth of $\bar{B}$. One finds that stability requires the two conditions
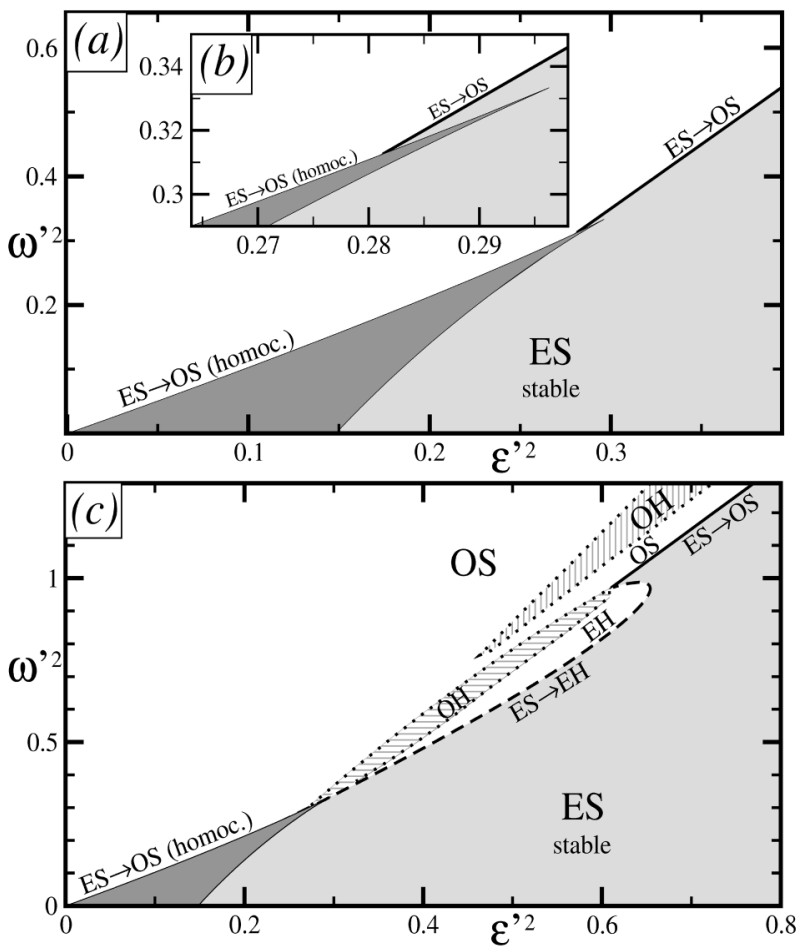

FIG. 3. Instabilities of the ES in the parameter space $\epsilon^{\prime 2}$ vs $\omega^{\prime 2}$ of the amplitude Eqs. (2). Inside the gray areas the ES are stable, and inside the dark gray region three solutions of Eq. (3) exist. In (a) and (b) we consider the $\bar{B}=0$ case. The thick straight line indicates the supercritical Hopf bifurcation ES $\rightarrow$ OS which appears for $\epsilon^{\prime 2}>0.281$ (for $\epsilon^{\prime 2}<0.281$ this transition occurs via a homoclinic bifurcation). In (c) all the primary instability lines of the ES are shown for $h^{\prime}=2.1$ and $\alpha^{\prime}=0.7$, for the complete Eqs. (2). Thick straight and dashed lines correspond to $\mathrm{ES} \rightarrow \mathrm{OS}$ and $\mathrm{ES} \rightarrow \mathrm{EH}$, respectively. Also, the secondary bifurcations (obtained numerically) of $\mathrm{OS}$ and $\mathrm{EH}$ leading to $\mathrm{OH}$ are shown (hatched regions enclosed by dotted lines).

$$
\begin{gathered}
h^{\prime} F^{2}-1>0, \\
\left(h^{\prime} F^{2}-1\right)^{2}+\omega^{\prime 2} / 4-\alpha^{\prime 2} F^{2}>0 .
\end{gathered}
$$

Breakdown of condition (4a) leads to a Hopf bifurcation with frequency $\Omega_{\mathrm{OH}}=\sqrt{\omega^{12} / 4-\alpha^{12} / 2}$ at $\epsilon_{\mathrm{OH}}^{\prime}=\left[\left(1 / h^{\prime}-1\right)^{2}+\omega^{\prime 2}\right] / h^{\prime}$. We note that, while the orbit in the $\bar{B}$ plane has a frequency $\Omega_{\mathrm{OH}}$, the one in the $\bar{A}$ plane has $2 \Omega_{\mathrm{OH}}$ and that a time shift of half a period transforms $(\bar{A}, \bar{B}) \rightarrow(\bar{A},-\bar{B})$. Consequently, this instability leads from entrained stripes to oscillating hexagons (ES $\rightarrow \mathrm{OH}$; see Fig. 4). This instability preempts the $\mathrm{ES} \rightarrow \mathrm{OS}$ bifurcation for $h^{\prime}<2$ and the opposite is true for $h^{\prime}>2$ [see Fig. 3(c)].

Breakdown of (4b) can arise as the first instability only when $\omega^{\prime 2}<\left(4 / h^{\prime}\right) \alpha^{\prime 2}$ if $h^{\prime}<2$ or $\omega^{\prime 2}<2 \alpha^{\prime 2}-\left(h^{\prime}-2\right)^{2}$ if $h^{\prime}>2$. This bifurcation is stationary and it leads to entrained hexagons (ES $\rightarrow \mathrm{EH}$; see Fig. 5) which occur in two symmetry degenerated variants related by $\bar{B} \rightarrow-\bar{B}$.

We now go beyond the thresholds of the primary transitions already described. Consequently, we resort to a numerical analysis of Eqs. (2). We will concentrate on the scenario relevant for the experiment and show that one may expect different kinds of hexagonal patterns in addition to the ones reported thus far.

For the experiments reported, $h^{\prime}$ should be larger than 2 such that the destabilization of entrained stripes with increasing $\omega^{\prime}$ leads to oscillating stripes $(\mathrm{ES} \rightarrow \mathrm{OS})$. Here we choose $h^{\prime}=2.1$ and $\alpha^{\prime}=0.7$. For this case, if we increase $\omega^{\prime}$ (as in the experiment) and for fixed $\epsilon^{\prime}$, not too small $\left(\epsilon^{\prime 2} \geqq 0.61\right.$ in this case), one first encounters the $\mathrm{ES} \rightarrow \mathrm{OS}$ [see Fig. 3(c)], next there is a supercritical bifurcation to oscillating hexagons. The resulting hexagons have the same symmetries as the ones encountered in the $\mathrm{ES} \rightarrow \mathrm{OH}$. Increasing $\omega^{\prime}$ further, there is a reen-
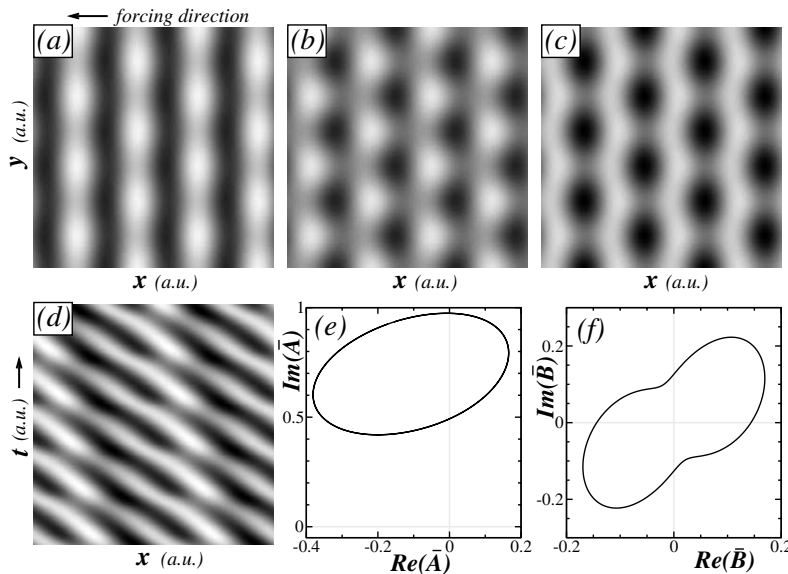

FIG. 4. Oscillating hexagons reconstructed from Eqs. (1) after the bifurcation given by condition (4a) for $h^{\prime}=1.95$, $\alpha^{\prime}=0.7, \epsilon^{\prime}=0.9$, and $\omega^{\prime}=1.2$. In (a), (b), and (c) three consecutive snapshots are shown. In (d) the space-time plot along a horizontal cut is shown. In (e) and (f) the limit cycle is shown in the planes $\bar{A}$ and $\bar{B}$. The term (a.u.) designates arbitrary units. 


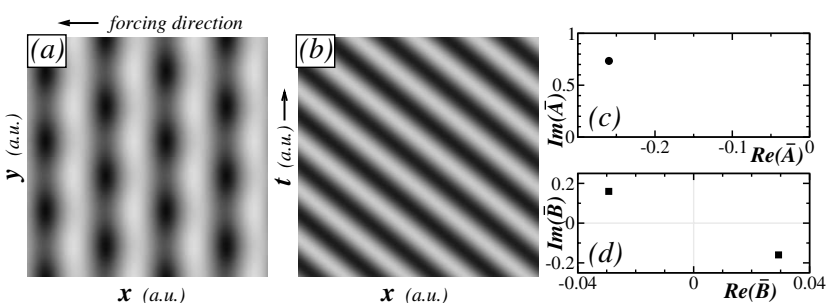

FIG. 5. Entrained hexagons reconstructed from Eqs. (1) after the $\mathrm{ES} \rightarrow \mathrm{EH}$ bifurcation for $h^{\prime}=2.1, \alpha^{\prime}=0.7, \epsilon^{\prime}=0.7$, and $\omega^{\prime}=0.83$ [cf. Equation (4b)]. In (a) and (b) we show a snapshot and its corresponding spatiotemporal plot. In (c) and (d) we show the location of the fixed point in the $\bar{A}$ and $\bar{B}$ planes, respectively.

trant transition where $\bar{B}$ vanishes leading back to oscillating stripes, which then persist up to arbitrary $\omega^{\prime}$.

For intermediate values of $\epsilon^{\prime}\left(0.296 \lesssim \epsilon^{2} \lesssim 0.61\right)$, as $\omega^{\prime}$ is increased, one typically encounters a supercritical Hopf bifurcation of the entrained hexagons. In these oscillating hexagons [also called $\mathrm{OH}$ in Fig. 3(c)], the $\bar{B}$ orbit has the same frequency as the $\bar{A}$ orbit, and we continue having two separate symmetry-degenerate solutions. As before, for high enough $\omega^{\prime}$ a transition to reentrant oscillating stripes takes place. Before this transition, more complex dynamics may occur, in particular, period doubling cascades to chaos. We have also observed gluing of chaotic, symmetry-degenerate attractors and ungluing. Finally, we point out that similar complex behavior is observed for slightly smaller values of $\epsilon^{\prime}$ $\left(\epsilon^{\prime 2} \lesssim 0.296\right)$, when the upper bound of the region of multiplicity (dark gray area in Fig. 3) is crossed.

Choosing $\alpha^{\prime}$ smaller than 0.7, the region where hexagons appear shrinks and finally vanishes. This occurs at $\alpha^{\prime} \sim 0.6$ for the oscillating hexagons at large $\epsilon^{\prime}$ and at $\alpha^{\prime} \sim 0.45$ for the entrained hexagons.

At the root of the phenomena presented is the competition between an anisotropic driving and the three-wave resonance mechanism leading to hexagons. Whereas one would naively expect that such a forcing would always increase the amplitude of the relevant stripe system and thus suppress the growth of the other modes compared to the unforced case, the situation is much richer. In fact, we are in the regime where the interaction of the modes forming $120^{\circ}$ is such that they tend to depress each other, thus leading to stripes rather than hexagons in the unforced case. On the other hand, here the amplitude of the forced stripe system is increased by the forcing only at low frequency, but decreased at high frequency [see Eq. (3)]. Therefore, at high frequency, the growth of the other two modes is enhanced compared to the unforced case. In particular, the modes leading to the hexagons are enhanced, unless $\alpha=0$. Then other states are presumed to arise, in particular, rectangular patterns.

Conclusions.-Here we report on the experimental discovery of the generation of a hexagonal symmetry by traveling-stripe forcing in Turing patterns. The origin of this phenomenon can be understood in terms of a shift of the transition boundary between stripes and hexagons. However, other dynamical behaviors such as different types of oscillating and chaotic hexagons have no counterpart in the unforced system. Thus, the introduction of a frequency via a spatial resonance mechanism has endowed the system with a wealth of novel and complex nonlinear dynamics which remains yet to be explored in depth. Finally, the very nature of the amplitude equation description guarantees the generic character of these results. While we have already observed the basic phenomenon in a simple model of the CDIMA reaction [12], we expect that similar phenomena should be found in a variety of systems undergoing the same type of stationary instability, from hydrodynamics to nonlinear optics or biophysics.

This work has been supported by the European Commission under network HPRN-CT-2002-00312 and DGI (Spain) under Projects No. BQU2003-05042 and No. BFM2000-0348. We thank S. Rüdiger and W. Pesch for useful discussions. Part of the simulations were done with the XDim Interactive Simulation Package developed by P. Coullet and M. Monticelli, who L. K. thanks for their support.

[1] V. Petrov, Q. Ouyang, and H. L. Swinney, Nature (London) 388, 655 (1997).

[2] A. Careta and F. Sagués, J. Chem. Phys. 92, 1098 (1990).

[3] A. K. Horvath, M. Dolnik, A.P. Muñuzuri, A. M. Zhabotinsky, and I. R. Epstein, Phys. Rev. Lett. 83, 2950 (1999).

[4] M. Lowe, J. P. Gollub, and T. C. Lubensky, Phys. Rev. Lett. 51, 786 (1983).

[5] P. Coullet, Phys. Rev. Lett. 56, 724 (1986).

[6] M. Dolnik, I. Berenstein, A. M. Zhabotinsky, and I. R. Epstein, Phys. Rev. Lett. 87, 238301 (2001).

[7] L. Yang, M. Dolnik, A. M. Zhabotinsky, and I. R. Epstein, Phys. Rev. Lett. 88, 208303 (2002).

[8] S. Rüdiger, D. G. Míguez, A. P. Muñuzuri, F. Sagués, and J. Casademunt, Phys. Rev. Lett. 90, 128301 (2003).

[9] V. Castets, E. Dulos, J. Boissonade, and P. De Kepper, Phys. Rev. Lett. 64, 2953 (1990); Q. Ouyang and H. L. Swinney, Nature (London) 352, 610 (1991).

[10] Chemical Waves and Patterns, edited by R. Kapral and K. Showalter (Kluwer Academic, Dordrecht, 1995).

[11] I. Lengyel, G. Rábai, and I. R. Epstein, J. Am. Chem. Soc. 112, 4606 (1990).

[12] A. P. Muñuzuri, M. Dolnik, A. M. Zhabotinsky, and I. R. Epstein, J. Am. Chem. Soc. 121, 8065 (1999).

[13] B. Peña, C. Pérez-García, A. Sanz-Anchelergues, D. G. Míguez, and A. P. Muñuzuri, Phys. Rev. E 68, 056206 (2003).

[14] M. Auer, F. H. Busse, and R. M. Clever, J. Fluid Mech. 301, 371 (1995); F. H. Busse, O. Brausch, M. Jaletzky, and W. Pesch, in Intermittency in Turbulent Flows, edited by J.C. Vassilikos (Cambridge University Press, Cambridge, England, 2000). 